\title{
INVESTIGACIÓN Y FORMACIÓN DE DOCENTES EN LA ESCUELA NORMAL SUPERIOR Análisis y perspectivas ${ }^{1}$
}

\begin{abstract}
Resumen
Los diversos documentos producidos y elaborados a propósito de la investigación por maestros y estudiantes de la ENSNSP durante los procesos de acreditación previa y de calidad, se constituyeron en fuentes para la reflexión alrededor de tres aspectos: El primero, el carácter y lugar de la Escuela Normal dentro del Sistema Educativo Colombiano en atención al marco político y normativo; el segundo, las posturas propuestas por los Centros de investigación, las facultades de educación y los Asesores y expertos, sobre lo que debía ser la investigación en estas instituciones formadoras de maestros; finalmente, los tipos de apropiación, sentidos y preguntas de investigación que daban cuenta de los ejercicios de investigación adelantados hasta el momento en la Escuela Normal. Estas reflexiones permitieron la construcción de una postura frente a la investigación que permitió redefinir la estructura curricular en la perspectiva del fortalecimiento de los procesos de formación inicial, complementaria y continuada de maestros en la institución.
\end{abstract}

Palabras clave. Escuela Normal Superior, maestros, estudiantes, investigación, facultades de educación. acreditación.

\section{Abstract}

The diverse documents produced and elaborated documents concerning to the teachers and students of the ENSNSP research during the previous accreditation and quality processes, were constituted in sources for the reflection around three aspects: The first one, the character and place of the Normal School inside the Colombian Educational System in attention to the political and normative mark; the second, the proposed postures by the Research Centers, the education schools, the advisers and experts, on what was due to be the research in these institutions former of teachers; finally, the appropriation types, senses and research questions that gave an account of the research exercises advance until the moment in the Normal School. These reflections allowed the construction of a posture in front of research that allowed to redefine the curricular structure in the perspective of the strengthening of the initial, complementary and continuous forming of teachers in the institution.

Key words. Normal High School, teachers, students, research, Education Schools, accreditation.

*Profesora de la Escuela Normal Superior de Nuestra Señora de la Paz. Coordinadora del Ciclo Complementario de Formación Docente. E-mail: azules@supercabletv.net.co

1 Este documento es parte de la investigación en el marco del convenio entre la Escuela Normal Superior Nuestra Señora de la Paz y la Sociedad Colombiana de Pedagogía. Fue presentado en el Congreso Internacional de Pedagogía, Bucaramanga, UIS-Escuela Normal Superior de Pie de Cuesta, octubre de 2003. Recibido el 30 de octubre de 2003 y aprobado el 14 de noviembre de 2003. 
Este documento es un abstract del informe preliminar de un trabajo de investigación que venimos realizando desde el año 2002, en compañía de la maestra Sandra Rodríguez ${ }^{2}$; en él damos cuenta de la experiencia de transformación en investigación y formación de maestros que ha tenido lugar en la ENSNSP ${ }^{3}$, a partir de la indagación de diversas fuentes de la memoria institucional, recopiladas desde el momento en el cual se inicia el proceso de Acreditación Previa, hasta cuando se termina la autoevaluación en el proceso de acreditación de calidad ${ }^{4}$. Nos propusimos tres reflexiones fundamentales: la primera, que implicó como tarea, hacer un reconocimiento del marco normativo y político desde el cual se adelantó el proceso de transformación institucional hacia "Escuela Normal Superior", nos permitió llegar a un análisis sobre el limbo jurídico, en el cual se encuentra el carácter y el lugar de estas instituciones en el sistema educativo colombiano.

La segunda reflexión, es un análisis de las diferentes posturas expresadas por centros de investigación, Facultades de Educación, asesores y expertos, con respecto a lo que las Escuelas Normales Superiores, deberían adelantar en el campo de la investigación educativa y pedagógica. La última reflexión, derivada de la anterior, consistió en mirar los tipos de apropiación, los sentidos y las preguntas, que han caracterizado los ejercicios de apro-

\footnotetext{
${ }^{2}$ Maestra encargada de orientar y coordinar los procesos de formación en investigación de los estudiantes y maestros de Ciclo Complementario de la Escuela Normal Superior Nuestra Señora de la Paz, en el marco del convenio que desde hace tres años, esta institución sostiene con la Sociedad Colombiana de Pedagogía (SOCOLPE).

${ }^{3}$ Escuela Normal Superior Nuestra Señora de la Paz.

${ }^{4}$ Hemos tomado este periodo de tiempo considerando que la Escuela Normal inició un nuevo proceso en su trayectoria institucional a partir de presupuestos normativos que le asignaron actividades hasta entonces adelantadas, fundamentalmente, por las Facultades de Educación y los Centros de Investigación.
}

ximación al trabajo investigativo adelantado en la ENSNSP; el trabajo de indagación se realizó acopiando la producción de maestros, estudiantes, directivos y colectivos de trabajo, que en distintos momentos se han hecho la pregunta por la investigación. A partir de un amplio espectro documental -actas, memorias, relatorías, documentos de trabajo, borradores, manuscritos, trabajos de grado, documentos institucionales, entre otros-, establecimos los momentos del proceso de lo que consideramos ha sido la construcción de nuestra postura sobre investigación educativa y pedagógica.

Dilucidar las aproximaciones sobre lo que implica investigar, es una acción que desencadena la pregunta por la formación. Por tal razón, lo que se inició como un proyecto de investigación sobre la investigación en la Escuela Normal, ha devenido en la redefinición de nuestra propuesta de formación de maestros, propendiendo por la articulación en los procesos de formación inicial, complementaria y continuada de maestros, con el propósito de desplegar la investigación en función del fortalecimiento académico de los diferentes niveles de formación docente. Este documento presenta, entonces, una breve reseña de las reflexiones realizadas y cierra con una presentación rápida de la actual propuesta de formación en la ENSNSP.

\section{CONTEXTO POLÍTICO Y NORMATIVO}

La normatividad educativa de la última década del siglo XX, transformó las condiciones en las cuales se adelanta la formación de docentes en el país, tanto en las Escuelas Normales como en las Facultades de Educación. Desde la Ley General de Educación (1994) hasta su Decreto reglamentario 1850 (2002), han aparecido principios y funciones que hacen más próximos estos dos escenarios de formación, al involucrar en el espacio de las Escuelas Normales actividades propias del

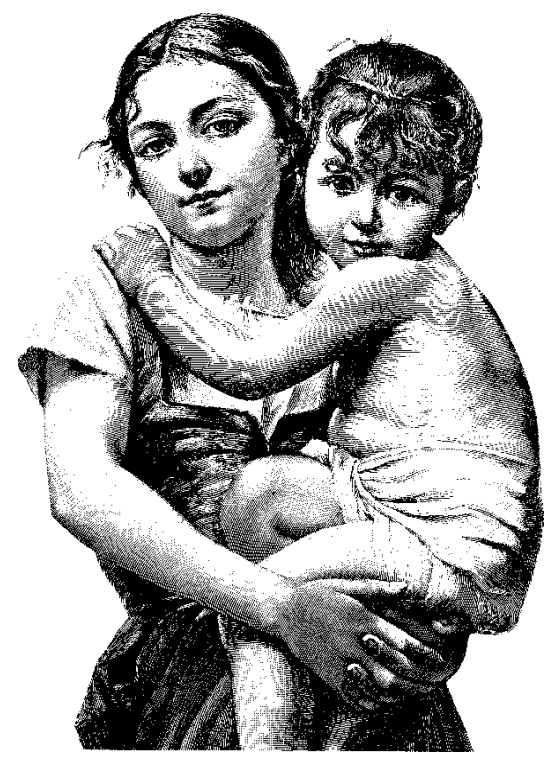

ámbito académico de la universidad como es el caso de la investigación.

En la revisión sobre el momento en el cual aparece la investigación como una de las actividades que deben adelantar las Escuelas Normales, el primer documento en el cual se les atribuye responsabilidad en la formación inicial de docentes funcionando como unidades de apoyo académico de instituciones de educación superior, es la Ley General de Educación ${ }^{5}$, que en el parágrafo del artículo 112 expresa: "estas instituciones debidamente reestructuradas y aprobadas estarían autorizadas para la formación de educadores de los niveles de preescolar y básica primaria, operando como unidades de apoyo académico para la formación inicial de docentes y mediante convenio con una institución de Educación Superior podrían ofrecer formación complementaria conducente al título de Normalista Superior". De esta manera, se obliga a las Escuelas Normales al cumplimiento de las finalidades de la formación de educadores, señaladas en el artículo 109 de la misma ley, una de las cuales propone: "el fortalecimiento de la inves-

\footnotetext{
${ }^{5}$ Ley 115 de febrero 8 de 1994
} 
tigación en el campo pedagógico y el saber específico", con lo cual parte del supuesto, de una trayectoria en investigación sobre lo pedagógico y sobre las disciplinas o saberes específicos que debe ser enriquecida, a partir del trabajo de las Escuelas Normales. Estos dos campos aparecen como objetos de investigación, sin aclarar su naturaleza.

Desde este momento, se hace visible en el discurso de los directivos de las instituciones formadoras de maestros, al igual que en el de los funcionarios del Ministerio de Educación y de algunas Secretarías de Educación, la idea de la investigación como acción indiscutible en las Escuelas Normales.

El entonces Ministro de Educación, Jaime Niño Díez, en las palabras de clausura del Tercer Encuentro de Investigación y Desarrollo Pedagógico en el Distrito Capital ${ }^{6}$, planteó que la política educativa propuesta por el Ministerio, buscaba convertir en hechos educativos las disposiciones surgidas de la Constitución Política, la Ley General de Educación y el Plan Decenal, llenando de contenido la descentralización, la participación y la autonomía escolar y universitaria, para lo cual, la investigación educativa debe tener como fin último, el "contribuir a la transformación de las prácticas formativas, a la creación de un nuevo colombiano y a participar activamente en el cambio social"; indica que hasta hace apenas treinta años se han venido dando los primeros pasos en investigación educativa en Colombia, siendo incipientes, pero significativos sus resultados; consideramos pertinente revisar el balance que allí se presentó, en el cual se valorarían otros alcances al reconocerle a la investigación educativa, una función diferente a la de darle sentido y contenido a las políticas y leyes educativas.

\footnotetext{
${ }^{6}$ Revista Educación y Ciudad N4, diciembre de 1997- enero de 1998. La nueva institucionalización de la Investigación Educativa", pp. 6-11.
}

En esta misma presentación, se planteaba que dentro de la política existían unas pautas organizacionales con las cuales se buscaría, entre otras cosas, "poner en funcionamiento las Normales Superiores y llevar acabo un profundo proceso de transformación de los programas universitarios de formación de profesionales de la educación, mediante la cualificación de las facultades de educación y su sometimiento a procesos de acreditación previa. Las Normales Superiores y las facultades de educación se verán abocadas a asumir nuevas prácticas formadoras, haciendo que cada cohorte de estudiantes constituya en sí misma un proyecto de investigación que se desarrolle dentro de teorías y prácticas que serán las mismas que le corresponderá aplicar al futuro profesional en su trabajo escolar".

En este sentido, se empiezan a vislumbrar unas primeras caracterizaciones para la investigación que le corresponde a las instituciones comprometidas en los procesos de formación de maestros; se llama la atención en la idea de hacer que cada cohorte de estudiantes se constituya en sí mismo en un proyecto de investigación, pues implica que esta "acción de conocimiento"7, no sea una tarea adicional de la Escuela Normal y de la facultad de educación, sino que haga parte de su naturaleza; por lo tanto, la propuesta curricular y el proyecto de formación se constituyen en un proyecto de investigación, lo que le permite movilidad, flexibilidad y le abre un campo de posibilidades hacia la experimentación, en la tarea de problematizar y responder permanentemente por los procesos de formación de maestros. Ni en este

\footnotetext{
${ }^{7}$ La idea de hablar de la investigación como "una acción de conocimiento problematizadora y sumativa, realizada en una tradición de saber acumulado y que cumple con los criterios de confiabilidad científica y social", corresponde a la propuesta que Olga Lucía Zuluaga presenta durante el Seminario de ASONEN acerca de los procesos de acreditación de Escuelas Normales, en marzo de 2001.
}

texto, ni en los que aparecen posteriormente, se hace diferenciación alguna entre la investigación que hacen las Escuelas Normales y la que hacen las Facultades de Educación.

En diciembre de 1997, el Ministerio de Educación Nacional presenta el Decreto 3012, por el cual se reglamentan las disposiciones para la organización y el funcionamiento de las Escuelas Normales Superiores, cumpliendo así con los artículos 112 y $216^{8}$ de la Ley General de Educación; en este decreto, las Escuelas normales quedan definidas como "unidades de apoyo académico", que deben atender la formación inicial de los educadores, para el ejercicio de la docencia en el nivel de preescolar y en el ciclo de educación básica primaria, sin aclarar el lugar en el cual se encuentra la Escuela Normal dentro del Sistema Nacional de Formación Docente y menos dentro del sistema educativo.

Por un lado, la formación docente inicial corresponde a educación media; por lo tanto, debe regirse dentro de los parámetros de la legislación general para este nivel, contemplados en la Ley 115 de 1994 y sus decretos reglamentarios; por otro lado, la formación complementaria tiene todas las características de educación superior y de hecho se organiza dentro de una normatividad diferente, entendiéndola como formación profesional de los educadores, según lo expresado en el artículo 2 del decreto 3012, acer-

\footnotetext{
${ }^{8}$ El artículo 216 plantea: "El Gobierno Nacional dentro del término de un (1) año contado a partir de la promulgación de la presente ley, determinará los procedimientos para reestructurar las normales que, por necesidad del servicio educativo, puedan formar educadores a nivel de normalistas superiores.

Las normales que no sean reestructuradas ajustarán sus programas para ofrecer, de acuerdo con lo dispuesto en el presente artículo, preferiblemente programas de la educación media técnica u otros de la educación por niveles y grados, según las necesidades regionales y locales.

La Nación y las entidades territoriales crearán las condiciones para dar cumplimiento a lo dispuesto en el presente artículo.
} 
ca de las finalidades de la Escuela Normal Superior. Este "limbo jurídico", además, obliga a las Escuelas Normales a dar cuenta de procesos de investigación y de desarrollo de planes de asistencia técnico-pedagógica para otras instituciones educativas.

La investigación en el ámbito de lo pedagógico y la investigación pedagógica, parecen constituirse en el objeto de la investigación de las Normales Superiores; esto se verifica en el artículo 2, literal b del decreto 3012 que indica como finalidad de la Escuela Normal: "desarrollar en los educadores en formación, las capacidades de la investigación pedagógica y de orientación y acompañamiento pedagógico de los educandos"; este articulado es complementado con el parágrafo del artículo 4: como parte de su proceso curricular, las Escuelas Normales Superiores deberán diseñar y ejecutar propuestas de investigación pedagógica y planes de asistencia técnico pedagógica para las instituciones educativas de su área de influencia".

Esta finalidad expresada en la Ley, implica una doble función de la investigación pedagógica en las Normales Superiores; por un lado, la formación y por el otro, la producción de saber pedagógico; con respecto a la primera función, requiere la participación de los estudiantes en procesos investigativos que les permita adquirir herramientas para la realización o participación en equipos de investigación; en la segunda, se trata de producir resultados y avances en los procesos investigativos, que contribuyan al mejoramiento y la innovación en prácticas y métodos pedagógicos. La doble función se anuda con la posibilidad de hacer de cada cohorte de estudiantes un proyecto de investigación, pues el proyecto de formación de maestros se constituye en la pregunta permanente de la institución y es desde ella que se hace posible su producción de saber en el campo pedagógico. Lo dicho hasta aquí guarda corres- pondencia con lo planteado en el literal f del artículo 13, donde se establece como objetivo del proceso de acreditación de las Normales Superiores "favorecer el sentido y la práctica de la investigación pedagógica".

Finalmente, es preciso reseñar el documento marco de acreditación ${ }^{9}$ en el cual la investigación aparece como referente de calidad, esto es, como "una unidad de análisis de carácter educativo que permite agrupar un conjunto de factores alrededor de un aspecto o conjunto de aspectos, de cuyo estado y comportamiento institucional depende que se aprecie o no la existencia de condiciones adecuadas para el logro de una educación de calidad en el marco del $P E I$ y de los requerimientos básicos derivados de las tendencias, investigaciones, avances y normas existentes al respecto ${ }^{10 "}$. Desde este lugar, la investigación se constituye en un escenario que da cuenta, del estado y las condiciones en términos de coherencia, identidad, eficacia, eficiencia, integridad y pertinencia de la propuesta académica y desde ella, de la fundamentación pedagógica y didáctica, de los actores, de la cultura y de la organización institucional.

Entonces, la investigación es un lugar de análisis privilegiado, desde el cual se hace posible mostrar el avance institucional, en términos de su responsabilidad, dentro del proceso que adelanta frente a la formación de maestros. Es importante citar que, en el documento marco, la investigación vuelve a ser propuesta como educativa, lo que amplia su campo; esto, si se considera lo educativo como un ámbito en el que lo pedagógico puede o no tener lugar; en este sentido, es preciso anotar que a lo largo de las normas y sus reglamentaciones, han aparecido diferentes referencias de lo que es

\footnotetext{
${ }^{9}$ Documento Marco. Serie Documentos Formación de Maestros: Ministerio de Educación Nacional. Bogotá, junio de 2000.

10 lbíd., p. 25.
}

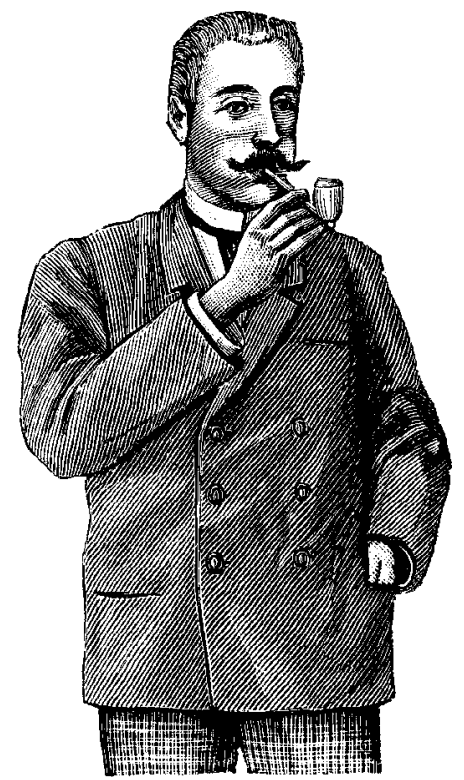

y debe ser la investigación en las instituciones formadoras de maestros, calificándola de diferentes maneras y exigiendo de ella unos resultados que, sin lugar a dudas, hablan de los distintos lugares de mirada que han tenido, quienes han producido dicha legislación, sobre el acto mismo de investigar, de sus objetos y los sujetos que han de participar y responder por ella.

Esta condición, unida a la diversidad de miradas presentadas por parte de expertos en el tema durante los diferentes encuentros organizados por ASONEN ${ }^{11}$, ASCOFA$\mathrm{DE}^{12}$, Ministerio de Educación $\mathrm{Na}$ cional y las propuestas particulares desarrolladas por cada Escuela Normal a partir de las asesorías ofrecidas por las Facultades de Educación con las cuales se estableció convenio, han trazado diversas perspectivas de lo que significa y le implica a una Escuela Normal hacer investigación. Por tanto, una revisión de la normatividad, de las políticas que han enmarcado estos procesos y de las diferentes perspectivas

\footnotetext{
11 Asociación Nacional de Escuelas Normales.

12 Asociación Colombina de Facultades de Educación.
} 
construidas desde diversas experiencias y encuentros, ha contribuido a fundamentar y construir una postura propia de la ENSNSP sobre la investigación educativa y pedagógica, que está en capacidad de adelantar.

\section{DE LAS DIVERSAS CONCEPCIONES SOBRE LA INVESTIGACIÓN EN LAS ESCUELAS NORMALES A LA CONSTRUCCIÓN DE UNA POSTURA SOBRE INVESTIGACIÓN}

Como se mencionó antes, una vez que las escuelas normales iniciaron sus procesos para alcanzar la acreditación previa y la acreditación de calidad y desarrollo, apareció una serie de miradas y primeras conceptualizaciones sobre lo que debería ser la investigación en estas instituciones, encargadas ahora de la formación inicial y complementaria docentes. Estas miradas fueron propuestas por expertos de las facultades de educación, especialmente de la Universidad Pedagógica Nacional y de la Universidad de Antioquia, quienes, a través de los eventos convocados por ASONEN, ASCOFADE o el Ministerio de Educación, presentaron a las escuelas normales documentos de trabajo y tareas concretas que les permitieran iniciar algunos ejercicios investigativos. Por otra parte, estas y otras facultades de educación, a través de los convenios exigidos por el $\mathrm{CAENS}^{13}$, para que las escuelas normales fueran acreditadas, han producido una variedad de textos que dan cuenta de posturas diferentes acerca de la investigación que le corresponde hoy a estas instituciones.

La recopilación de los documentos que han transitado en la ENSNSP,

\footnotetext{
${ }^{13}$ CAENS, Consejo Nacional de Acreditación para Escuelas Normales Superiores; instancia del Ministerio de Educación creada para los procesos de acreditación previa, en 1996, pero que dejó de funcionar durante la acreditación de calidad.
}

y su lectura trasversal para producir una matriz, en la que se presentan algunas categorías sobre las cuales se han estructurado las ideas de investigación propuestas, aparecen en el segundo informe de avance del proyecto de investigación que, para el efecto de esta presentación, no consideramos pertinente presentar pero que nos permitió describir y analizar las diferentes rutas que la Escuela Normal ha trazado en la construcción de una postura sobre la investigación educativa y pedagógica.

Este trazado se constituye de los aportes de varias instituciones y entidades y de las elaboraciones adelantadas por los equipos de docentes que han participado del proceso desde 1997; se realizó entonces, un análisis de las diferentes elaboraciones sobre investigación, de las consideraciones que se han hecho sobre líneas y proyectos, de las perspectivas que se han puesto en juego en los procesos de formación en investigación con estudiantes y maestros y de la configuración de los problemas que han sido objeto

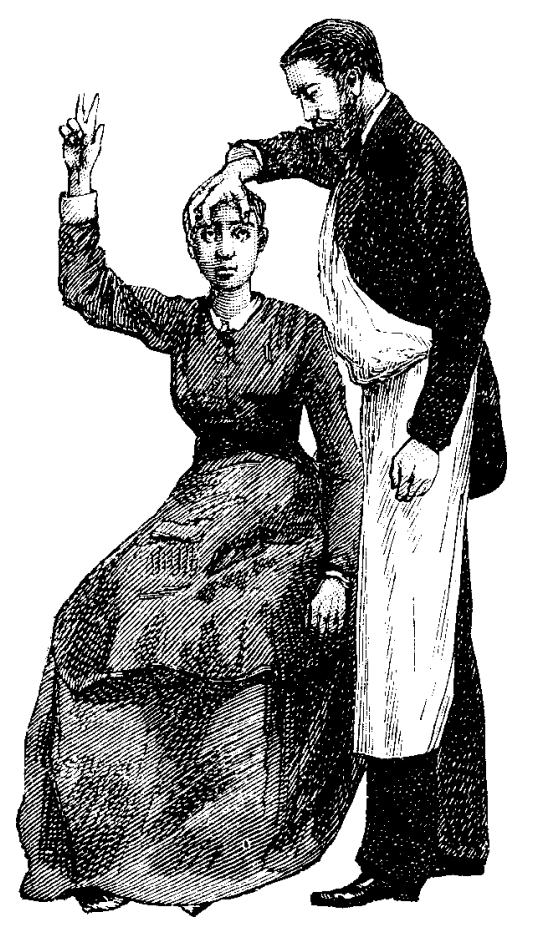

de reflexión, análisis y discusión de quienes han adelantado trabajos de investigación en la ENSNSP.

\subsection{Investigación: aproximaciones a su conceptualización en la escuela normal}

Se develaron las diferentes formas de entender la investigación, apropiadas 0 acuñadas por la ENSNSP, desde el momento en el cual se expresa, de manera explícita, un interés y una preocupación por adelantar procesos investigativos hasta la actualidad. En este recorrido se encuentran las siguientes acepciones sobre investigación:

1997. Investigación como actividad propia de las Escuelas Normales.

1997. Investigación como indagación sobre las cosas.

1997-1999. Investigación como campo de experimentación.

1999. Como objeto de estudio y de formación.

1999 .Como proceso de producción de saber.

1999/2003. Como proceso de reflexión y problematización sobre los supuestos educativos y pedagógicos. En este último momento, consideramos pertinente hacer énfasis, por las transformaciones e implicaciones que significa para el proyecto de la Escuela Normal, establecer un convenio con SOCOLPE ${ }^{14}$, para la ejecución del proyecto de investigación denominado, "Infancia, el Saber pedagógico y la escuela en la Sociedad Contemporánea".

\subsection{El proyecto de investigación en la escuela normal: desarrollos conceptuales}

El problema de investigación del proyecto, partió de la formulación de tres hipótesis sobre la educación contemporánea, construidas a propósito del trabajo de investigación adelantado por SOCOLPE: "La crisis actual de la escuela, la revalori-

\footnotetext{
14 Sociedad Colombiana de Pedagogía.
} 


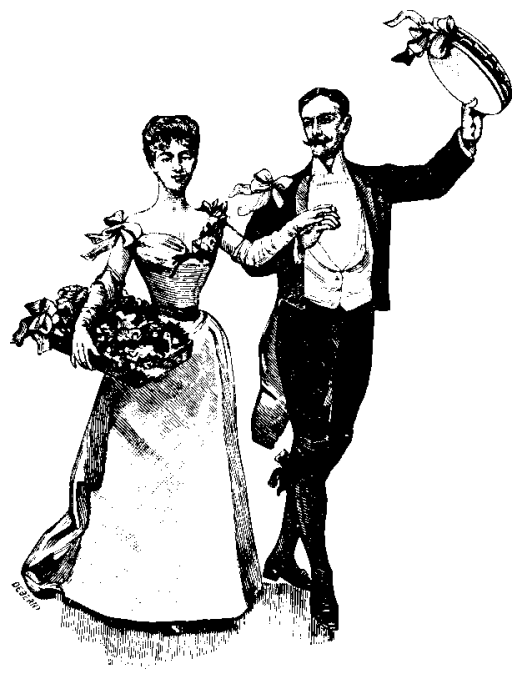

zación de la infancia y la tendencia hacia la desaparición de la infancia." Con respecto a la primera hipótesis de trabajo se muestra la manera cómo, en diferentes instancias, se está "llamando la atención sobre el papel de la institución escolar y su vigencia en el mundo actual (Tedesco: 1993; Puiggrós: 1994, CEPALUNESCO: 1992, PNUD-UNESCO: 1990, UNESCO: 1994, MEN: 1994, Misión de Ciencia, Educación y Desarrollo: 1994)"m5, poniendo en cuestión su pertinencia y legitimidad mediante la pregunta: "¿es aún necesaria la escuela?"16.

Esta decadencia de la escuela como institución privilegiada para la educación está indicando la aparición de otro acontecimiento educativo, "la Ciudad Educadora", en la cual "acontece de una manera mucho más compleja la producción y reproducción de los llamados códigos de la modernidad"17. La ciudad, en su condición de complejidad y de di-

\footnotetext{
${ }^{15}$ Noguera, Carlos y Rodríguez, Sandra. Proyecto de investigación institucional: Infancia, saber pedagógico y escuela en la sociedad contemporánea. ENSNSP-SOCOLPE, 2002.

${ }^{16} \mathrm{~A}$ propósito, ver el trabajo de investigación: Álvarez, Alejandro ... Y la escuela se hizo necesaria, Bogotá, SOCOLPE-Coop. Editorial Magisterio, 1996.

${ }_{17}$ Noguera, Carlos y Rodríguez, Sandra. Op. cit.
}

versidad, ha desplazado la escuela, pues no solamente se empodera desde una estrategia múltiple -escenarios educativos como agentes, contenidos y contextos-, sino que, además, desplaza la escuela como lo señalan recientes investigaciones ${ }^{18}$, en las cuales se señala "el vacío que la escuela le deja a los niños y jóvenes en la satisfacción de sus expectativas más sentidas" ${ }^{\prime \prime 9}$.

Sin embargo, paralela a esta mirada crítica sobre el papel de la escuela, también se presentan posiciones que sustentan su importancia y fijan el destino de las naciones en el aumento de la cobertura y la calidad de la educación escolar. Esta valoración por los procesos de escolarización, obedece a una responsabilidad compartida entre el Estado -cuya presencia cada vez es más tenue- y el sector privado, como se expresa en las políticas internacionales (políticas mundiales -Jomtien y CEPAL- y nacionales Salto Educativo, Plan para construir la paz y la Revolución educativa).

Lo que se deriva del problema formulado en el proyecto de investigación, es un cuestionamiento a la escuela por su condición en la actualidad, en tanto es una institución moderna y urbana que ya está entrando en decadencia en el mundo contemporáneo con la irrupción de las nuevas tecnologías y los efectos masivos de los medios. Aunque el documento inicial proponía una mirada histórica amplia sobre este problema (1960-1990), durante su desarrollo se ha propuesto posponer el ejercicio de indagación documental, para privilegiar la comprensión de los fenómenos contemporáneos, a partir de preguntas sobre la con-

\footnotetext{
${ }^{18}$ Ver los resultados del Proyecto ATLANTIDA: Adolescencia y Escuela, Bogotá: FES COLCIENCIAS-TERCER MUNDO, 1995, recogidos en cinco tomos titulados: La cultura fracturada, Todo lo que nos gusta se evapora, La ciudad nos habita, El silencio era una fiesta y Adolescentes colombianos ${ }_{19}$ Noguera, Carlos y Rodríguez, Sandra. Op. cit.
}

dición de época actual. Posteriormente, puede ser abordado este interés histórico, una vez la producción sobre la manera como se configura la educación contemporánea haya perfilado algunas problemáticas de las cuales se pueda llevar a cabo una aproximación genealógica.

En este sentido, el interés fundamental del proyecto con respecto a la escuela se ha centrado, por un lado, en indagar aquello que le está sucediendo, su proceso de deslegitimación y la forma como está transformando sus rituales, sus actores, sus métodos y su carácter. Por otro lado, estamos dando cuenta "de la emergencia de aquellas prácticas y escenarios educativos que caracterizan la Bogotá de hoy, de tal manera que podamos pensar, desde lo educativo, un nuevo modo de ser ciudadanos"20.

En lo que respecta a la segunda hipótesis, en el proyecto se muestra la manera cómo a principios de siglo se propuso una revalorización de la infancia basada en un profundo optimismo pedagógico. De ahí la insistencia de la sociedad en "la protección de la infancia y en la necesidad de transformar la educación infantil en las escuelas y la actitud de los padres frente a la educación de sus hijos"21. Este proceso de invención de la infancia implicó tres estrategias diferentes: la puesta en evidencia, por parte de ciertos intelectuales, del valor económico de la niñez, la necesidad de convencer a las madres europeas de la necesidad de amamantar a sus hijos y la consolidación del matrimonio en torno al amor y valoración de la relación marital.

La última hipótesis de trabajo, es uno de los planteamientos que ha generado mayor interés en la ENSNSP; esto se evidencia tanto en los documentos de los docen-

\footnotetext{
${ }^{20}$ Noguera, Carlos y Rodríguez, Sandra. Op. cit.

${ }_{21}$ lbid.
} 
tes, los ejercicios de los estudiantes, el mismo proyecto, como en los desarrollos propios que ha tenido la investigación. Es claro que no se trata sólo de un asunto académico e intelectual, sino de la aparición de un conjunto de prácticas y condiciones sociales, culturales y sobre todo, económicas, que están destituyendo la infancia; tal como lo afirma Postman, N. (1999), estamos asistiendo a la imposibilidad de dos situaciones: el control de la información y la secuencia en el aprendizaje que constituyeron y precavieron por mucho tiempo la infancia y que en la actualidad, han sido desdibujados por las recomposiciones que la comunicación y la información han sufrido en la sociedad a lo largo del siglo XX.

Estudios recientes, abordados por la ENSNSP, como el de Narodowski, M (1999), consideran que la sociedad del siglo XXI ha entrado, definitivamente, en un proceso de "desinfantilización", para lo cual señala dos rasgos particulares: la infancia hiperrealizada ${ }^{22}$ y la infancia desrealizada ${ }^{23}$. Estas dos nociones están siendo abordadas por estudiantes de la Escuela Normal Superior, me-

${ }^{22}$ Narodowsky, Mariano. 1999. Después de Clase. Los desencantos de la escuela actual. Buenos Aires. Novedades Educativas. "chicos que realizan su infancia con Internet, computadoras, sesenta y cinco canales de cable, vídeo, family games, y que hace ya mucho tiempo dejaron de ocupar el lugar del no saber", p. 47.

${ }^{23} \mathrm{Ibid}$. Se refiere a la infancia de la calle, abandonada, independiente, que trabaja desde muy temprana edad. "Son también los chicos y las chicas de la noche, que pudieron reconstruir una serie de códigos que les brindan cierta autonomía económica y cultural y les permiten realizarse, mejor dicho, des-realizarse, esa es la palabra correcta, como infancia. Son niños hacia los cuales difícilmente tendremos un sentimiento moderno de infancia, ternura y protección. Hay una niñez que no está infantilizada, una niñez que no es obediente -porque no precisa obedecer, en muchos casos-, una niñez que no es dependiente -es independiente en la negociación cotidiana para lograr su sustento- $y$, por tanto, una niñez que es autónoma -y que en la calle construye sus propias categorías morales-. Una niñez que, al verla sola o en grupo, difícilmente nos causa ternura", p. 51 . diante el desarrollo de varios subproyecto que se entretejen con este proyecto institucional, a través de las propuestas de investigación de los maestros en cada uno de los núcleos.

Al plantear estas condiciones de constitución de los niños contemporáneos, el proyecto derivó las siguientes preguntas que viene siendo objeto de reflexión y análisis en los seminarios de investigación con estudiantes y en las jornadas de trabajo del colectivo de maestros que se encuentran en el proceso de investigación formativa: "¿a quiénes pretendemos educar? ¿desde qué instituciones y procesos pretendemos hacerlo? ¿desde qué principios y con cuáles herramientas pretendemos hacerlo? Por ahora, como parece evidente, seguimos empeñados en una concepción de infancia que se estrella cada vez de manera más fuerte con esas nuevas subjetividades que habitan nuestros "jardines", escuelas y colegios"24.

\subsection{El proyecto de investigación en la escuela normal: desarrollos metodológicos}

En este sentido, la Escuela Normal hace una apuesta de formación en investigación, tanto para los maestros en ejercicio, como para los maestros en formación inicial y complementaria; en ambos casos viene desarrollando seminarios que favorecen la preparación de los maestros y la formulación de proyectos de investigación articulados al proyecto general propuesto.

Los estudiantes de Ciclo Complementario, dentro de cada uno de los núcleos de formación: Educación, sociedad y cultura, Prácticas pedagógicas y lenguajes, reciben orientación a propósito del proyecto de investigación que adelantan los maestros del núcleo, quienes, a su vez asisten al seminario de investi-

${ }^{24}$ Noguera, Carlos y Rodríguez, Sandra. Op. cit. gación formativa ${ }^{25}$ que se adelanta desde el 2002 hasta hoy y que busca evidenciar y discutir las posturas desplegadas en el trabajo de formación docente, construir una oferta curricular con mayores niveles de integración para el ciclo complementario y potenciar los procesos de investigación formativa, mediante la formulación y desarrollo de propuestas de investigación que se deriven y apoyen el desarrollo del proyecto que sirve de marco.

Cada proyecto de investigación de núcleo, tiene en cuenta los planteamientos centrales del proyecto global: infancia, saber pedagógico y escuela en la sociedad contemporánea, por lo cual, se tienen tres modalidades de trabajo: lectura y discusión de documentos, video-foros y análisis de la experiencia docente. Como resultado de este proceso de discusión se perfilaron tres campos de trabajo que, en la actualidad, se encuentran en curso: discusión y actualización, articulación de la propuesta curricular y proceso de investigación.

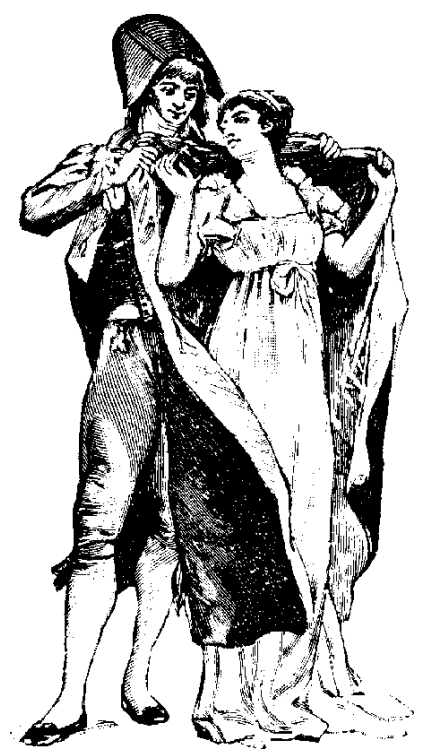

${ }^{25}$ Estos seminarios desarrollados con maestros y estudiantes adelantados del CCFD, son recogidos en una relatoría que insertamos en el segundo informe de investigación, para explicar los desarrollos y el estado actual del seminario. 


\section{PROPUESTA DE FORMACIÓN DE DOCENTES EN Y PARA LA PAZ}

Tal como lo señala el proyecto de investigación, estamos asistiendo al cuestionamiento de una serie de supuestos desde los cuales hemos adelantado las prácticas de formación de docentes. En este caso, se podrían señalar como supuestos fundamentales: el carácter teóricopráctico de la profesión docente; la coherencia entre el ámbito teórico -entendido como el campo de la reflexión- con el ámbito de la práctica -entendido como el campo de la aplicación- y la institución escolar como escenario privilegiado para el ejercicio profesional.

Al enunciar estos supuestos no se expresan desacuerdos substanciales sobre su pertinencia en los programas de formación, y cuando se discuten, se pone el acento en mejorarlos y no en problematizarlos. Al enunciar estos mismos presupuestos como formulaciones que pueden ser cuestionadas, redireccionadas e incluso desmitificadas como presupuestos de la formación de los docentes, el análisis abandona el ámbito de lo deseable (acercamiento entre la teoría y la práctica), para instalarse en el ámbito de lo posible (configuración de la práctica docente como un campo de tensiones). En la ENSNSP, hemos considerado que abordar estas preguntas como objetos de la reflexión y de la formación, permite que quienes estamos participando de esta experiencia pedagógica, nos preguntemos por el mundo contemporáneo en función de desplegar una de las expresiones del carisma de la congregación "formación de docentes para la paz", en las condiciones de época actuales.

Al problematizar estos supuestos, estamos proponiendo un análisis sobre los componentes constitutivos de la formación docente -teoría y práctica-, no en la perspectiva lineal y secuencial de la formación -primero la teoría, luego la práctica- o en el orden de la aplicabilidad -la teoría aplicada en la práctica, ya sea en el proceso secuencial o de manera simultánea-, sino en su condición tensional. Una mirada a la formación alejada de una relación causal en la cual la fundamentación lleva a la aplicación, impide entender las condición de realización del ejercicio donde ocurren relaciones de carácter tensional, en las cuales se apuesta por la constitución de subjetividades y por la legitimación de posicionamientos discursivos que configuran el campo educativo ${ }^{26}$.

Esta perspectiva de la formación, tensional y problematizadora, permite que el maestro se involucre en este proceso y se piense en una sociedad globalizada y neoliberal, no para acceder a ella o ser producido por ella, sino para tomar postura y decidir desde ella, a partir de las condiciones de diversidad y multiplicidad que le posibilite su formación.

\subsection{Formación inicial de docentes}

La estructura curricular para educación media, tiene en cuenta tres aspectos: fundamentación en la ley 115 de 1994 y sus decretos reglamentarios; énfasis y áreas optativas correspondientes a la naturaleza de la escuela normal; dinámica académica y organizacional que se corresponda con la propuesta para la formación complementaria docente.

La educación media en la ENSNSP es de carácter académica, las áreas obligatorias y fundamentales se encuentran organizadas en tres núcleos: sociedad, educación y cultura; matemáticas y ciencias naturales, y lenguajes; la intención es que se trabaje de manera articulada alrededor de temáticas, para que al entrar en contacto con los otros núcleos se puedan desarrollar las propuestas interdisciplinares (proyectos

\footnotetext{
${ }^{26}$ Rodríguez, Sandra. La práctica pedagógica: un campo de tensiones. En prensa. 2002. Conjeturas, Universidad Distrital.
}

pedagógicos de naturaleza problematizadora). Las áreas optativas se organizan en un núcleo de prácticas pedagógicas, que incluye además de la experiencia y la aproximación a procesos pedagógicos y educativos, unos primeros acercamientos a ejercicios de investigación. El énfasis en lenguajes y la formación en investigación se desarrollan de otra forma a partir de las propuestas interdisciplinares y seminarios.

\subsection{Formación complementaria de docentes}

La propuesta curricular para el $\mathrm{Ci}$ clo Complementario hoy se encuentra en tránsito: desde la estructura existente entre 1999 y 2002, hasta la diseñada y organizada a partir de los resultados de esta investigación y de las continuas reflexiones del colectivo docente de la ENSNSP, que se proyecta alcanzar en el 2005.

La primera estructura responde al afán de cumplir con los requerimientos organizacionales, propios de los niveles que han sustentado la vida escolar; entonces, se encuentra una organización por campos, áreas de formación y núcleos que terminan fragmentados en asignaturas; vale la pena aclarar aquí, que esta fragmentación, además, se ha constituido en requisito, que las facultades de educación plantean para hacer posible el reconocimiento de los estudios adelantados por los normalistas superiores, en términos sólo de homologación de asignaturas y no del título alcanzado en la Escuela Normal; por otro lado, la propuesta hacia la que vamos caminando, se encuentra organizada desde la misma intuición de campos y áreas de formación, con un reajuste en los núcleos, en la organización y administración de los tiempos y espacios tanto de maestros como de estudiantes.

Los núcleos propuestos para el primer año tienen una naturaleza temática, constituyéndose en los ambiente académicos donde se hace posible la fundamentación y la discusión de teorías y modelos alrede- 
dor de las categorías que anudan los núcleos. La discusión de los equipos docentes en cada uno de los núcleos, además de las diferentes experiencias que los estudiantes tienen en su desarrollo, dan lugar a la formulación de preguntas de investigación. Estas preguntas, desde su formulación pasando por su desarrollo teórico, ofrecen la posibilidad de conversar, debatir y aprender durante los seminarios del primer año; además, dan lugar a la formulación de los núcleos problémicos, para el trabajo de segundo año. Es importante destacar que en esta propuesta los proyectos de práctica pedagógica están anudados a los desarrollos de cada uno de los núcleos, según su naturaleza y pertinencia; en este último sentido, se destaca el valor de la experimentación alrededor de las tendencias educativas y pedagógicas actuales. Durante el segundo año, en el trabajo por núcleos, se continua en su dinámica de trabajo, pero se plantea el desarrollo de discusiones y la orientación de tareas propias de las preguntas que se formularon para la investigación durante el primer año.

Esta es, pues, una descripción breve, de la comprensión y construcción que en la ENSNSP se ha alcanzado, en el ejercicio de pensarse como institución formadora de

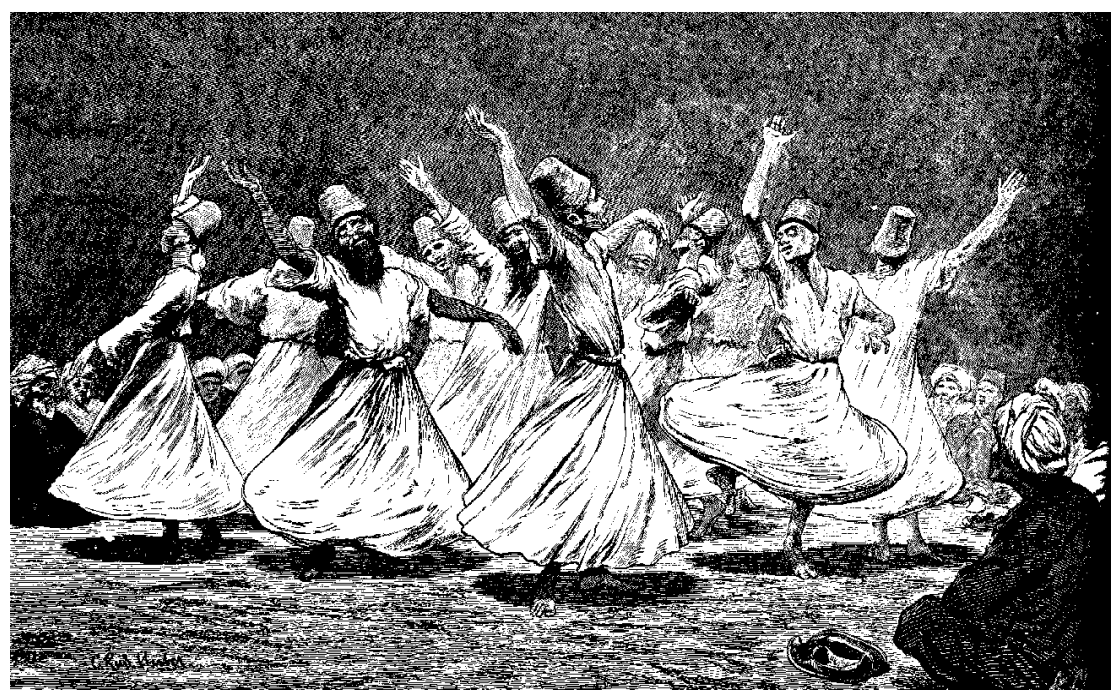

maestros y maestras en y para la paz, como es su tarea congregacional. Esperamos que las diferentes opiniones y argumentos expuestos aquí, sirvan y contribuyan a la apertura de un verdadero debate sobre el ámbito educativo y pedagógico en investigación y formación de maestros.

\section{Bibliografía}

Álvarez G., Alejandro. "... Y la escuela se hizo necesaria", Bogotá, Cooperativa Editorial Magisterio, 1995.

. "La historia de la escuela en Colombia, El siglo XIX", Bogotá, UPTC, 1990.

"El pensamiento histórico narrativo y el proyecto civilizador". Bogotá, UPN, 1991.

BADINTER, Elisabeth. ¿Existe el instinto maternal? Historia del amor maternal. Siglos XVII al XX, Barcelona, Paidós, 1991

Bourdieu, Pierre. "Campo intelectual y proyecto creador". En: Problemas de estructuralismo, México, Siglo XXI, 1971, pp. 135-172.

y PASseron, Jean Claude. "La re-

CARLI, Sandra. Infancia y Sociedad, Buenos Aires, Galerna, 1991. producción", Barcelona, Laia, 1977.
CAStRo, Jorge Orlando. República que sufre mano ociosa está achacosa, Bogotá, CIUP-UPN, 1991.

"Hospicios, escuelas y panópticos". Ponencia presentada al Primer Congreso Iberoamericano de Docentes e Investigadores en Historia de la Educación Latinoamericana, Bogotá, septiembre de 1992.

Educación pública y reforma educacionista en Colombia, Bogotá, CIUPUPN, 1990

CEPAL-UNESCO: Educación y conocimiento: eje de la transformación productiva con equidad. Santiago de Chile, 1992.

CONFERENCIA IBEROAMERICANADEMINISTROS DE EDUCACIÓN. Declaración de los Ministros de Educación Iberoamericana en Salvador. Salvador, Bahía-Brasil. 7 y 8 de julio de 1993.

DíAz, Mario. "Pedagogía, discurso y poder". En: Pedagogía, Discurso y Poder. Bogotá, CORPRODIC, 1990, pp. 39-67.

"Campo intelectual de la educación". Cali: Textos Universitarios - Universidad del Valle, 1994.

ECHEVERRI, Alberto. Santander y la instrucción pública. Bogotá, FORO NAL. POR COLOMBIA-Universidad de Antioquia, 1989.

Foucault, Michel y otros. Espacios de Poder. Madrid, La Piqueta, 1991.

Vigilar y castigar: nacimiento de la prisión, 11. México, Siglo XXI, 1985.

GIROux, Henry. "Teorías de la reproducción y la resistencia en la nueva sociología de la educación: una análisis crítico". En: Revista Colombiana de Educación, № 17, Bogotá, CIUP, 1986.

HeLg, Aline. La educación en Colombia: 1918-1957. Bogotá, CEREC, 1987.

ILLICH, Iván, y otros. Educación sin escuelas. Barcelona, Península, 1975.

\section{La sociedad desescolarizada.} Barcelona, Barral, 1974.

Martínez, Alberto; Castro, Jorge Orlando; Noguera, Carlos Ernesto. Escuela, maestro y vida cotidiana en Santafé colonial. Bogotá, Socolpe, 2000. 
MINISTERIO DE EDUCACIÓN NACIONAL. Lineamientos generales de procesos curriculares. Hacia la construcción de comunidades educativas autónomas -Documento 1-. Santa Fe de Bogotá: MEN, 1994.

MISIÓNDE CIENCIAEDUCACIÓNYDESARROLLO. Al filo de la oportunidad. Santa Fe de Bogotá: Presidencia de la república, 1994.

NARODOWSKI, Mariano. La utilización de periodizaciones maropolíticas en historia de la educación. Algunos problemas. Buenos Aires, NEES, 1994.

Después de clase. Desencantos y desafíos de la escuela actual, Buenos Aires, Ediciones Novedades Educativas, 1999.

Noguera, Carlos Ernesto. Vida, raza, escuela. Bogotá, CIUP-UPN, 1990 (Mec.).

, Álvarez, Alejandro; Castro, Jorge Orlando. La ciudad como espacio educativo: Bogotá y Medellín durante la primera mitad del siglo XX. Bogotá, Arango Editores, Socolpe, 1999

PNUD-UNESCO: Conferencia Mundial sobre educación para todos. Satisfacción de las necesidades básicas de aprendizaje. Jomtien, 1990.

Postman, Neil. O desaparecimento da infância, Rio de Janeiro, Graphia, 1999.

PuIGgros, Adriana. "La táctica escolar". En: Sujetos, disciplina y currículum en el ori- gen del sistema educativo argentino, tomo I. Buenos Aires, Galerna, 1990.

. Imaginación y crisis en la educación latinoamericana. Buenos Aires, Rei Argentina S.A. - Instituto de Estudios y Acción Social, 1994.

. "Los anarquistas también enseñan". En: Sujetos, disciplina y currículum en el origen del sistema educativo argentino, tomo I. Buenos Aires, Galerna, 1990.

y otros. Estudios comparados en educación en América Latina. Buenos Aires, Grupo editor S.R.L., 1992.

"Los inmigrantes como educadores y las sociedades populares de educación". En: Sujetos, disciplina y currículum en el origen del sistema educativo argentino, tomo I. Buenos Aires, Galerna, 1990.

"Sujeto pedagógico y control social". En: Sujetos, disciplina y currículum en el origen del sistema educativo argentino, tomo I. Buenos Aires, Galerna, 1990.

América Latina: Crisis y prospectiva de la educación, Rei Argentina S.A., 1992.

Historia de la educación en la Argentina, t. 2, Buenos Aires, Galerna, 1991.

RAMA, G. "El estilo de modernización social. Primera etapa”. En: Educación, participa- ción y estilos de desarrollo en América Latina. Buenos Aires, Kapeluz, 1984.

REIMER, Everett, La escuela ha muerto. Barcelona, Barral, 1973.

Robles, Marta. Educacion y sociedad en la historia de México. México, siglo XXI, 1978.

Rockwell, Elsie y Mercado, Ruth. La escuela, lugar de trabajo docente. (Cuadernos de educación, descripciones y debates). México, DIE, 1986.

y ESPELETA, Justa. La escuela, relato de un proceso de construcción inconcluso. Documentos. México, DIE, 1986.

Tedesco, Juan Carlos. "Educación y sociedad en América Latina: algunos cambios conceptuales y políticos". En: Revista Colombiana de Educación. CIUP-UPN. Santa Fe de Bogotá, 1993, pp. 71-86.

"Educación y sociedad en la Argentina". En: Educación y sociedad en la Argentina (1880-1900). Buenos Aires, Capítulo, Centro Editor, 1982.

UNESCO: Proyecto 2000. La declaraciónPropuestas de actividades. París, 1994.

WeInBerg, G. "Hacia la educación popular". En: Modelos educativos en la historia de América Latina. Buenos Aires, Kapeluz, 1984.

ZANOTTI, L. "La escuela redentora de la humanidad". En: Etapas históricas de la política educativa. Buenos Aires, EUDEBA, 1972.

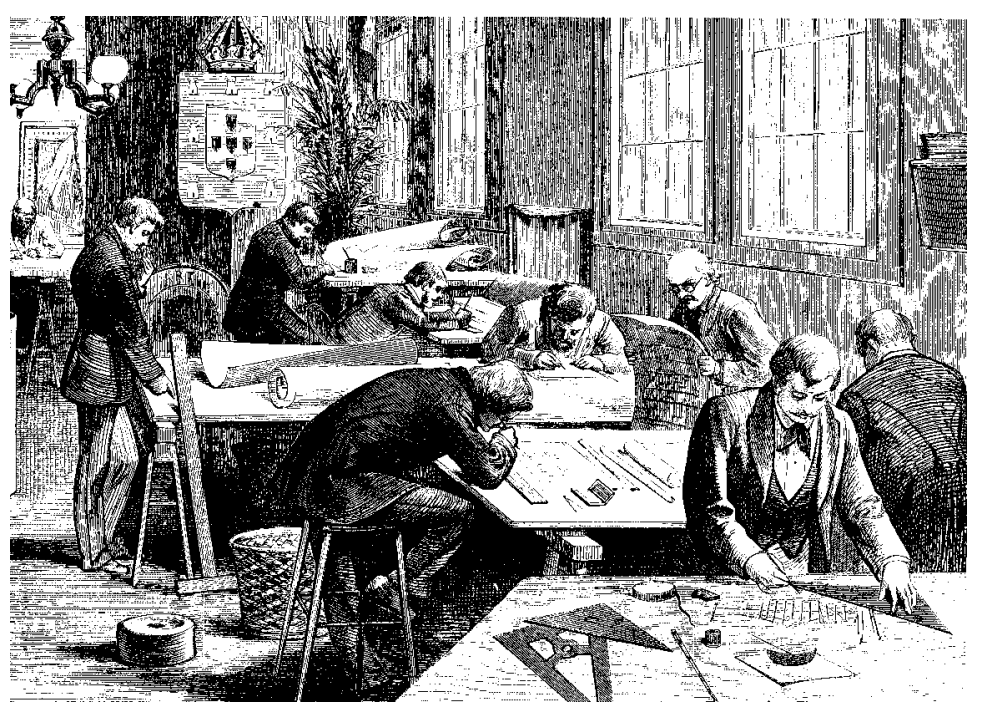

